\title{
Full characterization and comparison of phase properties of narrow linewidth lasers operating in the C-band
}

\author{
Radan Slavík ${ }^{1}$, Yi Liao ${ }^{1}$, Ed Austin ${ }^{2}$, Periklis Petropoulos ${ }^{1}$, and David J. Richardson ${ }^{1}$ \\ ${ }^{1}$ Optoelectronics Research Centre, University of Southampton, Southampton, SO17 1BJ, UK \\ Tel.:+44-2380594524 Fax+44-2380593142,e-mail:r.slavik@soton.ac.uk \\ ${ }^{2}$ Stingray Geophysical Limited, Guildford, Surrey, GU2 7YG, UK
}

\begin{abstract}
We characterize and compare the performance of various commercially available lasers in terms of their absolute frequency stability, lineshape and linewidth, and frequency noise. The frequency stability, linewidth and lineshape are evaluated using an 'optical ruler' - a carrier-envelope stabilized optical comb. The frequency noise is measured over an extended spectral range starting from $2 \mathrm{~Hz}$. The performed analysis gives data necessary when deciding which laser to use in a particular application.
\end{abstract}

Phase noise measurement, Lasers for Interferometric sensors

\section{Introduction}

Exploitation of the phase characteristics of optical signals is of ever increasing technological importance. This includes accurate control and measurement of phase in ultrasensitive interferometer-based component testing ${ }^{1}$, ultrahigh resolution optical spectroscopy ${ }^{2}$, and interferometric optical sensors ${ }^{3}$. Further, it includes also optical communications where the use of optical phase for encoding data attracts an increasing interest ${ }^{4}$. Within all of the above applications the absolute frequency stability, spectral linewidth, and spectrally/temporally dependent phase/frequency noise characteristics of the lasers used is critical to understanding/optimizing the system performance. Although various well-established techniques for measuring such source properties exist, it is becoming ever more challenging to properly characterize modern compact, high performance single frequency lasers since they are becoming ever quieter and boast ever narrower linewidths and absolute frequency stabilities. However, the best choice of laser for a given application can only be made when its detailed noise properties are known, and these depend on the cavity design, implementation and gain medium employed.

Here, we compare four compact commercially-available narrow-linewidth lasers emitting in the C-band based on different technologies using a number of state-of-the-art measurement techniques. Specifically, we measure the performance of a fiber-based Fabry-Perot laser (a ROCK laser module from NPhotonics Inc.), a distributed feedback fiber laser (a Basik module E15 from NKT Inc.), a hybrid integrated-optics:semiconductor laser (a RIO ORION module from Redfern Inc.), and a virtual-ring waveguide laser (Orbit laser from Orbits Lighwave, Inc.).

\section{Principles of Measurement}

Laser phase/frequency noise may be measured by heterodyning the laser with a reference laser, or directly at the optical frequency. The heterodyning technique uses two lasers that are simultaneously launched onto a fast photodiode, to produce a radio-frequency beat note at the difference frequency. The frequency fluctuations apparent on the beat note are the combination of the individual frequency fluctuations of the two lasers. (A single laser can also be superimposed with itself via a long fiber delay loop, but such self-homodyne systems are not suited for measuring laser frequency noise at low frequencies since impractically long delays are required). In our study, we beat the laser under test with an individual comb line of an Optical Frequency Comb (FC1500-250 Frequency synthesizer from Menlo Systems GmBH) employing carrier- 
envelope phase stabilization and locked to a Rubidium based clock which provides an absolute line-stability of better than 1 $\mathrm{kHz}$ - far better than the frequency stability of the lasers under test. We show two sets of data: (i) the central beat signal frequency variation measured each $1 \mathrm{~s}$ for a period of one hour (to measure the carrier frequency stability); and (ii) the beat signal noise averaged over $\sim 100 \mathrm{~ms}$ (to measure the linewidth/lineshape). Unfortunately, this method only allowed us to measure the noise up to a frequency of several $\mathrm{MHz}$ (limited by intensity noise of the measured lasers). Furthermore, this method does not provide any information about the spectral composition, and hence origin, of the frequency noise.

To increase the measured frequency range and to obtain the frequency noise spectrum we implemented a method based on the use of an optical frequency discriminator to convert frequency modulation to intensity modulation and which directly measures the noise at the optical frequency. We used a delayed Mach-Zehnder (MZ) interferometer ${ }^{5}$ as our discriminator and the principle of the method is outlined in Fig. 1. The interferometer is set to quadrature at which point any frequency variation at the input produces the largest variation in amplitude at the output (Fig. 1b). It is advantageous to use both interferometer outputs and to perform balanced detection since this generates a two times larger signal and allows the Relative Intensity Noise (RIN) contribution to the measured signal to be suppressed (see Fig. 1b). The interferometer delay must be chosen carefully: a large delay results in a larger measured signal since the slope of the MZ transfer function scales with the delay, however, a larger delay also narrows the bandwidth over which the interferometer transfer function is linear, which corresponds roughly to $1 / 3$ of the interferometer spectral period (as shown in Fig. 1b). This is generally sufficient only when the $1 / \mathrm{f}$ noise is dominant such that the white noise can be considered insignificant. Here, we use a much larger delay than that corresponding to the 1/f noise linewidth (to capture, e.g., white noise characteristics), as suggested in Fig. 1c. In this situation, it is necessary to compensate for the non-linear frequency response of the interferometer through appropriate data processing routines.

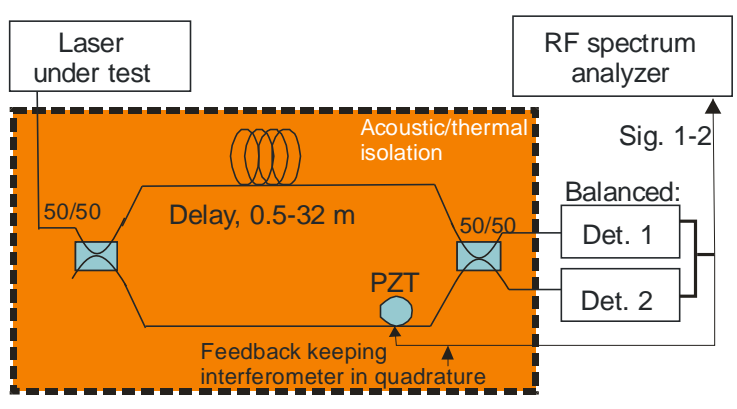

(a)

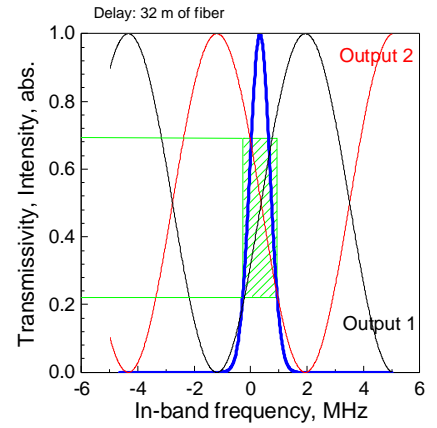

(b)

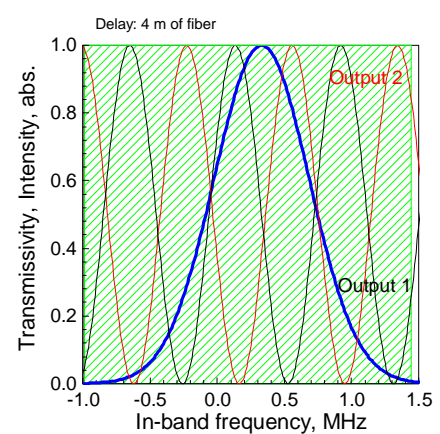

(c)

Fig. 1 Set-up for frequency noise measurement using a delayed MZ interferometer (a). Generally, only the linear part of the transfer function (red and black curves) is used (blue: laser under test) (b). Here, we use the full transfer characteristics (c) and correct for this in the data analysis.

\section{Results}

First, we measured the long term carrier frequency stability, (see Fig. 2). The lasers were each attached to a metallic laboratory table and the temperature was controlled via the laboratory air conditioning (giving a peak-to-peak temperature difference of $\pm 1 \mathrm{~K}$ for each laser). The lasers were fully exposed to the air draft from the air conditioning system. All lasers showed periodic behavior, suggesting that the surrounding temperature (periodically adjusted by bursts of cooling from the air conditioning) is the largest source of the drift. The RIO laser has a considerably smaller package and hence thermal mass than the others and this may explain is sensitivity to external temperature variations. The Orbit laser lacked the active temperature stabilization option - with this option, the manufacturer promises $< \pm 0.25 \mathrm{MHz}$ of the drift as opposed to $< \pm 20 \mathrm{MHz}$ when this option is not used. 


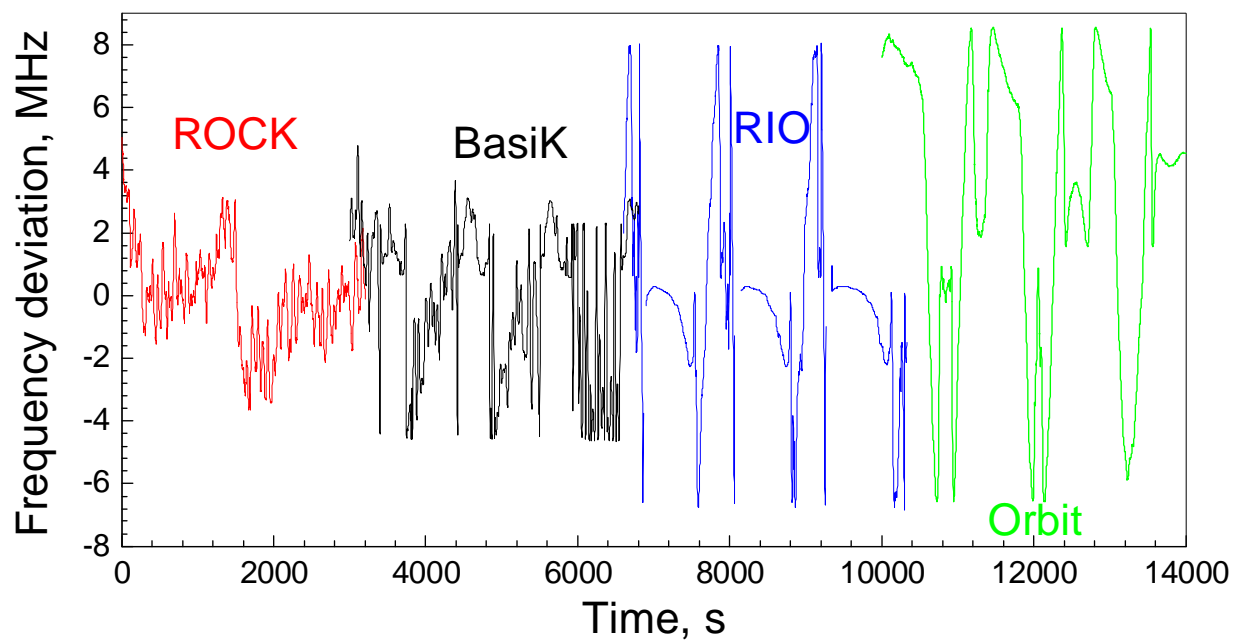

Fig 2 Deviation of the carrier frequency of the selected laser from the nearest comb line measured in an air conditioned laboratory with temperature stability of about $\pm 1 \mathrm{~K}$.

The results of the heterodyne beating measurement averaged over a $100 \mathrm{~ms}$ time interval are shown in Fig. 3 on both log and linear scales. The non-semiconductor lasers (the Rock, Orbit, and Basik) exhibit a Gaussian-like line shape, while the semiconductor-based RIO has a more Lorentzian-line shape. Note however that the RIO laser gave the best linewidth value when measured in this way (see Fig. 3). The spectrally-resolved frequency noise characteristics measured using the imbalanced interferometer are shown in Fig. 4. To obtain these results, different interferometer delays (in the range 0.5-32 m) were used and the data were stitched together. Note that special care was taken to obtain a signal that was at least $5 \mathrm{~dB}$ above the measurement noise floor (detector, spectrum analyzer, or RIN limited). The narrow peaks in the characteristics of the Orbit laser are - according to the manufacturer - caused by the faulty laser driver. At the conference, we expect presenting updated data using next-generation laser driver.
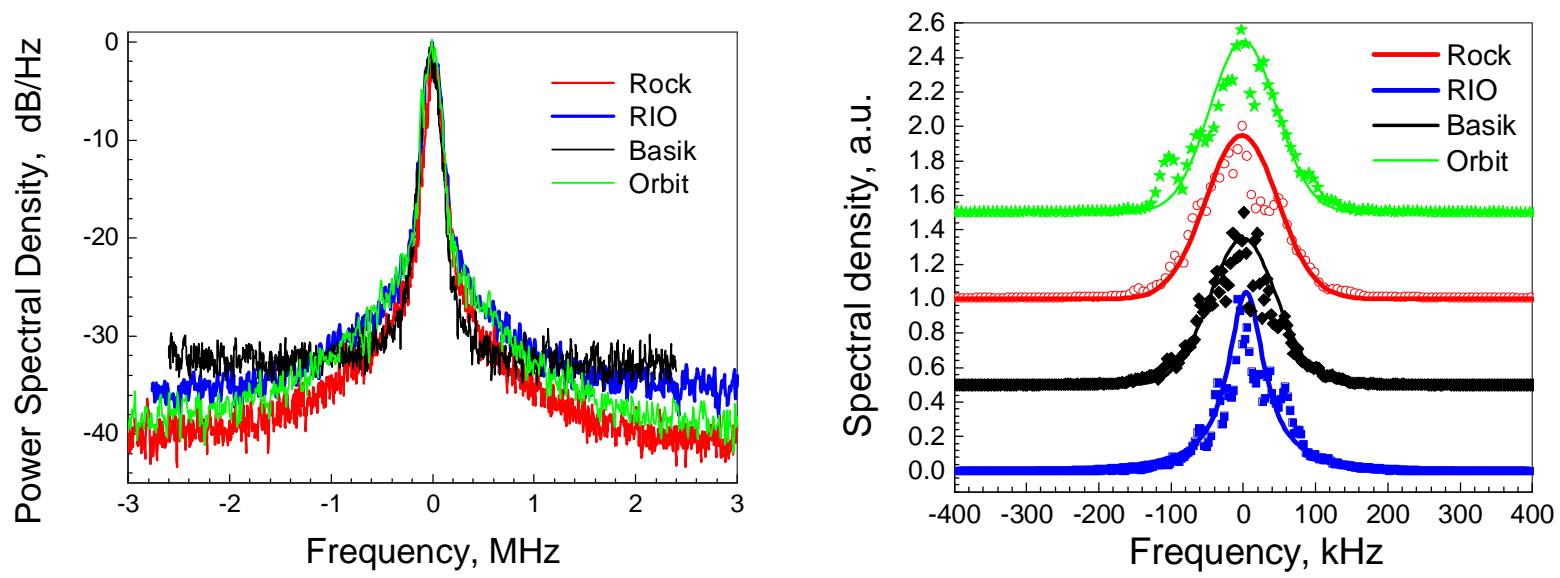

Fig. 3 Heterodyne signal averaged over $100 \mathrm{~ms}$ on a log scale (left) and on a linear scale with a peak fit giving a FWHM spectral width of $95 \mathrm{kHz}$ for the Orbit (Gauss fit), $120 \mathrm{kHz}$ for the Rock (Gauss fit), $60 \mathrm{kHz}$ for the RIO (Lorentz fit), and $95 \mathrm{kHz}$ for the Basik (Gaussian fit). All measured with a resolution of $6 \mathrm{kHz}$. 


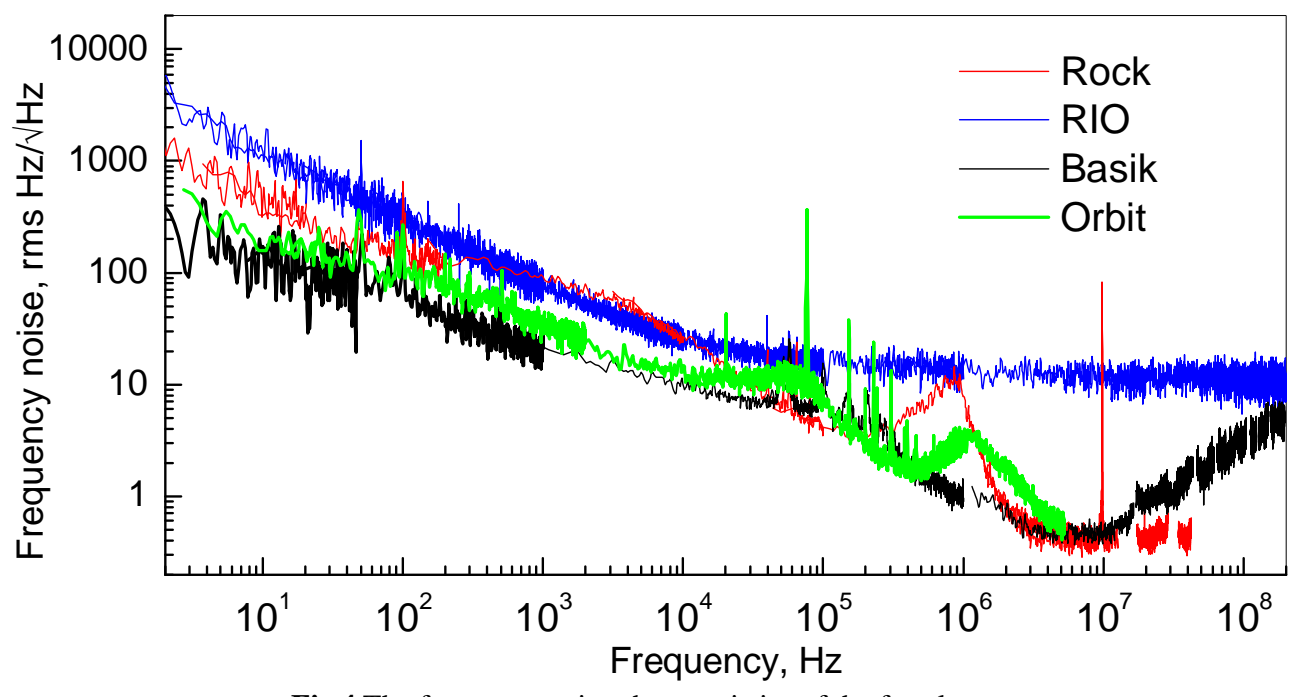

Fig 4 The frequency noise characteristics of the four lasers.

\section{Conclusions}

From our measurements we find that the Rock laser gives the best absolute wavelength stability (7 MHz peak-to-peak). Orbit laser with optional temperature stabilization is supposed to give even better stability, however it was not available for our tests. Rock and Orbit have the lowest frequency noise at frequencies above $5 \mathrm{MHz}$ (below noise floor of our set-up). By contrast the BasiK laser offers the lowest frequency noise at low frequencies (up to $40 \mathrm{kHz}$ ), but suffers from relatively high frequency noise in the $\mathrm{GHz}$ range. The RIO laser was found to exhibit a very narrow heterodyne linewidth of $60 \mathrm{kHz}$ and has no peaks in its frequency noise spectrum (unlike other), which we believe to be a consequence of the fact it does not incorporate any active electronic-based stabilization. However, we note that the RIO laser appears the most sensitive to temperature perturbations. Orbit laser exhibited noise characteristics slightly worse as compared to Basik at the low frequencies, but outperforms it at high frequencies.

This research has received funding in part from the UK Technology Strategy Board (FOSAR Deep) and the European Communities Seventh Framework Programme FP/2007-2013 under grant agreement 224547 (PHASORS). Useful discussions with Mr G. Marra of National Physical Laboratories are gratefully acknowledged.

\section{References}

1. www.lunatechnologies.com.

2. Inaba, H., Ikegami, T., Hong, F.-L., Bitou, Y., Onae, A., Schibli, T.R., Minoshima, K., and Matsumoto, M., "DopplerFree Spectroscopy Using a Continuous-Wave Optical Frequency Synthesizer," Appl. Opt. 45 4910-4915 (2006).

3. Dandridge, A. and Tveten, A.B., "Phase Noise of Single-Mode Diode Lasers in Interferometer Systems," Appl. Phys. Lett. 39 530-532 (1981).

4. Gnauck A.H. and Winzer P.J., Optical Phase-Shift-Keyed Transmission, J. of Lightwave Technol. 23 115-130 (2005).

5. Meng, Z., Hu, Y., Xiong, S., Stewaqrt, G., Whitenett, G., and Culshaw, B., "Phase Nose Characteristics of a DiodePumped Nd:YAG Laser in an Unbalanced Fiber-Optic Interferometer," Appl. Opt. 44 3425-3428 (2005). 